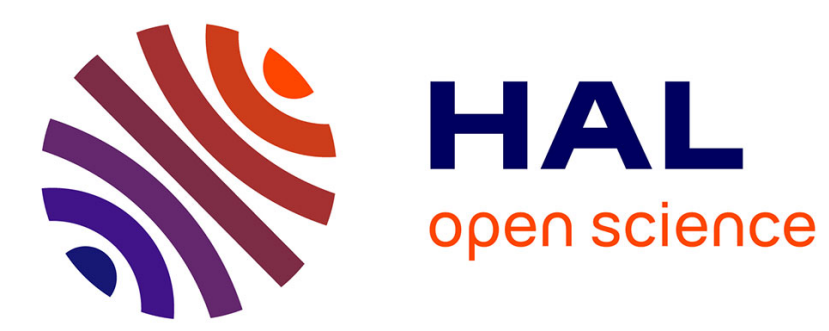

\title{
La centauresse, un exemple de remise en question de la frontière du genre à l'époque impériale
}

\author{
Eléonore Montbel
}

\section{To cite this version:}

Eléonore Montbel. La centauresse, un exemple de remise en question de la frontière du genre à l'époque impériale. Frontière $\times \mathrm{s}$ : revue d'archéologie, histoire et histoire de l'art, 2020, 2, 10.35562/frontieres.233 . hal-03063783

\section{HAL Id: hal-03063783 \\ https://hal.univ-lyon2.fr/hal-03063783}

Submitted on 14 Dec 2020

HAL is a multi-disciplinary open access archive for the deposit and dissemination of scientific research documents, whether they are published or not. The documents may come from teaching and research institutions in France or abroad, or from public or private research centers.
L'archive ouverte pluridisciplinaire HAL, est destinée au dépôt et à la diffusion de documents scientifiques de niveau recherche, publiés ou non, émanant des établissements d'enseignement et de recherche français ou étrangers, des laboratoires publics ou privés. 


\title{
Frontière's \\ Revue d'archéologie, histoire et histoire de l'art
}

\author{
Publiée avec le soutien de \\ la Maison de L'Orient et de la Méditerranée Jean Pouilloux \\ dans le cadre de la pépinière de revue PraIRIAL
}

\section{No 2 \\ Imaginer la frontière}

dir. Fabien BIÈVRE-PERRIN et Vincent CHOLLIER 


\section{Frontière $\cdot s$}

Maison de l'Orient et de la Méditerranée

$5 / 7$ rue Raulin

69365 LYON CEDEX 07

ISSN 2534-7535

frontiere-s@mom.fr

Pour soumettre un article et consulter l'appel en cours :

www.frontiere-s.mom.fr

https://publications-prairial.fr/frontiere-s

\section{Directrice des publications}

Françoise LE MORT, directrice de la MOM

Rédacteurs en chef

Fabien BIĖVRE-PERRIN (Centre Jean Bérard)

Vincent CHOLLIER (HiSoMA)

Gaëlle PERROT (HiSoMA)

Comité de rédaction

Loubna AYEB (Archéorient)

Mathilde DURIEZ (ArAr)

Cécile MOULIN (HiSoMA, ArAr)

Secrétaire de rédaction

Vincent CHOLLIER (HiSoMA)

Direction du numéro

Fabien BIÈVRE-PERRIN (Centre Jean Bérard) et Vincent CHOLLIER (HiSoMA)

Auteurs

Eléonore MONTBEL (Aix-Marseille Université, IRAA USR 3155)

Giovanni POLIZZI (Aix-Marseille Université, Centre Camille Jullian UMR 7299) et Samuel ROMEO (Università degli Studi di Palermo)

Benjamin Eldon STEVENS (Trinity University)

Vanessa RODRIGUES (ITEM - Identités, Territoires, Expressions, Mobilités EA 3002)

Photo de couverture :

Skyphos dit «Skyphos aux centaures », Berthouville, v. 50 apr. J. C. Paris, Bibliothèque nationale de France, inv. 56.6.

Source : https://commons.wikimedia.org/wiki/File:Cup_centaurs_Berthouville_CdM_n2.jpg

(Marie-Lan Nguyen, CC BY 2.5) 


\section{Sommaire du numéro}

LA CENTAURESSE, UN EXEMPLE DE REMISE EN QUESTION DE LA FRONTIÈRE DU GENRE À L'ÉPOQUE IMPÉRIALE

Eléonore Montbel

ENTRE DESTRUCTIONS ET RECONSTRUCTIONS, LE PATRIMOINE SICILIEN PENDANT ET APRÈS LA SECONDE GUERRE MONDIALE

Le cas de Solonte

Giovanni Polizzi et Samuel Romeo

RÉFLEXIONS CRITIQUES AUTOUR DES FRONTIÈRES DE LA PÉNINSULE

IBÉRIQUE AU PREMIER ÂGE DU FER

Vanessa Rodrigues

"Not the Lover's Choice, but the Poet's": Classical Receptions in

Portrait of a LADY ON Fire

Portrait de la jeune fille en feu, dir. Céline Sciamma, 2019

Benjamin Eldon Stevens

LES FRONTIÈRES DE L'AFRICA : ENTRETIEN AVEC STÉPHANIE GUÉDON

Stéphanie Guédon, Vincent Chollier et Gaëlle Perrot

COMPTE-RENDU

Agut-Labordère D. et Redon B. (éd.), Les vaisseaux du désert et des steppes

Nicolas Morand 



\title{
La centauresse, un exemple de remise en question de la frontière du genre à l'époque impériale
}

DOI : 10.35562/frontieres.233

\section{Eléonore Montbel}

\author{
Doctorante contractuelle, Aix-Marseille Université, CNRS, IRAA, Aix-en-Provence, France
}

\begin{abstract}
L'existence de types sexuels (mâle, femelle) chez une espèce suscite une frontière biologique commune à toute espèce vivante qui connaît une reproduction sexuée. Or lorsqu'il s'agit d'êtres mythiques ce système n'a pas de nécessité : dans l'imaginaire grec ancien il existe des groupes uniquement masculins ou féminins, tels que les sirènes, les satyres ou les centaures. Ces derniers, dans la Grèce archaïque et classique, sont uniquement des mâles et ont un caractère agressif et bestial, surtout envers les femmes. Pourtant la frontière du genre est un jour franchie et des parèdres voient le jour, parfois même accompagnées des petits centaures, témoignant d'une génération. Ces déclinaisons au féminin des centaures sont attestées dans la figuration à partir du er $^{e}$ siècle av. J.C., surtout en contextes domestique et funéraire. Elles apparaissent ainsi en « supplément 》 de leurs homologues masculins : elles sont plus tardives, sont représentées en nombre bien inférieur, et sont quasi absentes de la littérature - contrairement à eux. Il est question dans cet article de comprendre, à partir de la documentation antique figurée et écrite, pourquoi les centauresses sont absentes du monde grec et pourquoi a-t-on ressenti le besoin, au Ir $^{\text {r }}$ siècle av. J.C., de dépasser l'imaginaire établi et de créer une contrepartie de l'autre sexe.
\end{abstract}

Mots-clés : Antiquité, religion gréco-romaine, polythéisme, genre, mythologie, iconographie, être mythique, centaure, centauresse

The existence of sexual types (male, female) in a species creates a biological boundary common to any living species undergoing sexual reproduction. However, when it comes to mythical beings, this system has no necessity: in archaic and classic Greek imagination there are only male or female groups, such as sirens, satyrs or centaurs. However, the frontier of the genre is one day crossed and feminine counterpart are born, sometimes even accompanied by little centaurs, allowing a generation. These feminine variations of centaurs are attested in the figuration from the $I^{\text {st }} c . B C$, especially in domestic and funerary contexts. They thus appear as a "supplement" to their male counterparts : they appear later, are represented in much smaller numbers and are almost absent in literature - unlike them. This article aims to understand, from ancient figurative and written documentation, why centauresses are absent in the Greek world and why it was felt necessary in the $I^{\text {st }} c$. BC to go beyond the established imaginary and create a counterpart of the other sex.

Keywords: Antiquity, Greco-Roman religion, polytheism, gender, mythology, iconography, mythical being, centaur, centauress

L'Antiquité gréco-romaine a fourni de nombreux groupes de personnages fabuleux d'un seul sexe, comme les sirènes, les sphinx ${ }^{1}$, les satyres, les centaures. Ces espèces mythiques, à mi-chemin entre le réel et l'irréel, paraissent se borner à cette frontière du genre qu'elles ne dépassent, pour certaines, jamais. Êtres hybrides pouvant être appelés mixanthrôpoi ${ }^{2}$, ils se situent à la frontière de l'animal et de l'humain, limite retranscrite dans la figuration par un assemblage empruntant à plusieurs espèces. À l'époque archaïque et jusqu'au

1 Jusqu'au $\mathrm{VI}^{\mathrm{e}}$ siècle av. J.-C. uniquement, les sphinx sont parfois représentées avec une barbe : Tsiafakis 2003 , p. 75 , p. 80.

2 Le terme mixanthrôpos apparaît au IV ${ }^{\mathrm{e}}$ siècle et désigne des créatures issues d'un mélange entre l'homme et une autre espèce, se référer à Aston 2011. 
$\mathrm{V}^{\mathrm{e}}$ siècle av. J.C., la partie animale des centaures (exprimée par la violence) prend le dessus, notamment à travers la centauromachie ${ }^{3}$. Mais la porosité de la frontière humain/animal permit, au même moment, que des centaures plus humains voient le jour, tels que Pholos ou Chiron, à qui l'éducation de jeunes hommes sera confiée. C'est peutêtre à cause de ce caractère humain déjà présent que les centaures ont été l'un des rares hybrides dotés de parèdres, de manière ponctuelle au $\mathrm{v}^{\mathrm{e}}$ siècle av. J.C., plus nettement durant l'époque impériale. La Famille de centaures de Zeuxis (tableau décrit par Lucien au $\mathrm{II}^{\mathrm{e}}$ siècle apr. J.-C.) est le plus ancien exemple connu d'une famille de centaures, incluant une femelle et une progéniture. Par l'originalité de son sujet, cette œuvre témoigne de la variabilité des mythes grecs, dont les poètes qui les transmettent ne sauraient répondre à un dogme et ainsi restreindre leur activité fondamentalement créatrice ${ }^{4}$. C'est ce dont témoigne également Ovide, au

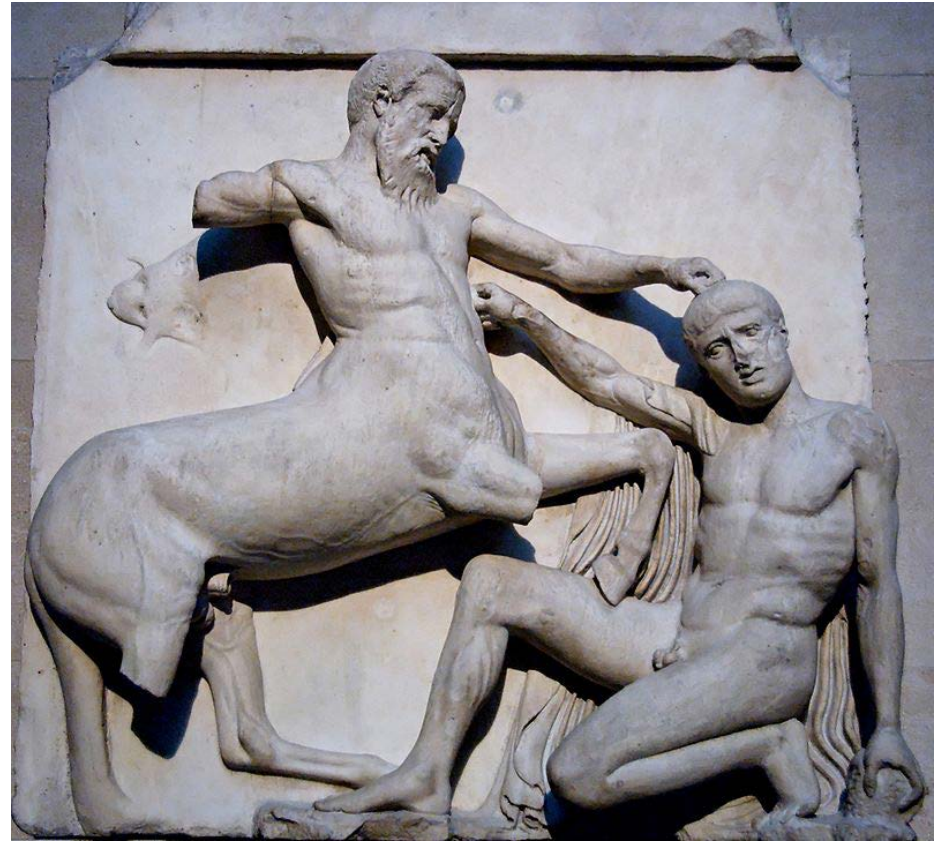

Figure 1. Métope sud (n 30) du Parthénon, 447-438 av. J.-C.

Londres, British Museum, inv. 1816,0610.14. Source : https:// commons.wikimedia.org/wiki/File:South_metope_30 Parthenon_BM.jpg (Marie-Lan Nguyen, domaine public) $\mathrm{I}^{\text {er }}$ siècle apr. J.C., lorsqu'il introduit le couple de centaures Cyllare et Hylonomé dans la centauromachie thessalienne ${ }^{5}$.

Sont présentés ici les résultats d'un travail effectué dans le cadre d'un mémoire de master, qui consistait en un recensement et une étude des attestations textuelles et figurées de centauresses dans l'Antiquité gréco-romaine. Au total, une soixantaine de représentations ont été relevées dans le monde grec et en Italie, parmi lesquelles seulement une dizaine datent des époques classique et hellénistique. Il est question dans cet article de comprendre comment le personnage de la centauresse, discret dans le monde grec, participe à partir de la fin de la République et à l'époque impériale à l'évolution de la figure mythique du centaure, passant de monstre stérile à hybride dompté, voire humanisé.

\section{DISCRÉTION DE LA CENTAURESSE DANS LE MONDE GREC}

\section{Le centaure grec}

Les premières images de centaures dans le monde grec apparaissent à l'époque archaïque et sont d'emblée empreintes d'un caractère violent et belliqueux. Impuissants face à la mania que déclenchent chez eux le vin et l'éros, ils constituent un ennemi idéal dans les récits hérö̈ques, particulièrement pour Héraclès qui les combat à deux reprises : une première fois lorsque le héros et le centaure Pholos partagent du vin, ce qui fait tomber la troupe dans une folie incontrôlable ${ }^{6}$; une seconde fois lorsque Nessos, faisant mine d'aider Déjanire à traverser l'Événos, tente de la violer ${ }^{7}$. Ces récits montrent que le rapport des centaures aux femmes est agressif. Ce constat est corroboré par un autre mythe célèbre, celui du combat contre les Lapithes, dont l'élément déclencheur est la tentative d'agression d'un centaure contre Hippodamie, la mariée ${ }^{8}$. Ces légendes définissent les centaures dans l'imaginaire grec archaïque et classique, les faisant apparaître comme des êtres agressifs et sauvages. $\mathrm{Au} \mathrm{VI}^{\mathrm{e}}$ siècle et plus encore à partir du $\mathrm{V}^{\mathrm{e}}$ siècle à cause des Guerres médiques,

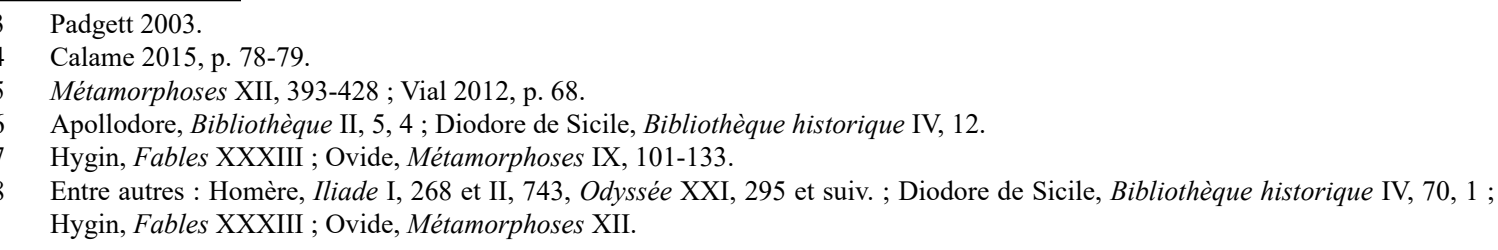


les centauromachies connaissent un vif succès dans les représentations figurées, notamment sur la céramique grecque puis dans le décor de bâtiments publics 9 . À cette époque, les ennemis mythiques mis en scène dans ces représentations figurées signifient, de manière métaphorique, la supériorité des Grecs sur les peuples barbares, en particulier les Perses. C'est ce qu'illustre le décor architectural du Parthénon à Athènes (fig. 1), où la centauromachie thessalienne (métopes sud), la gigantomachie (métopes est) et l'amazonomachie (métopes ouest) symbolisent la victoire d'Athènes sur la barbarie, incarnée ici par plusieurs évocations des forces du chaos. Plus généralement, les centaures sont aussi présents dans la décoration des temples postérieurs aux Guerres médiques, où ils incarnent l'Autre incivilisé ${ }^{10}$.

L'absence de parèdre chez les centaures s'explique, car la légende ne leur en a jamais fourni. Nés de l'accouplement entre un homme et une cavale, ils sont un prodige de l'ordre du monstrueux et n'ont pas vocation à avoir une descendance, encore moins une famille. La violence et l'agressivité ne sont d'ailleurs pas réservées aux êtres mythiques masculins, puisqu'on trouve ces caractéristiques chez les sphinx ou encore les sirènes qui vivent elles aussi sans parèdre et entretiennent un rapport malveillant avec le masculin ${ }^{11}$.

\section{La Famille de centaures de Zeuxis}

La première figuration d'une centauresse est sans doute due au peintre Zeuxis, à la fin du $\mathrm{v}^{\mathrm{e}}$ siècle av. J.-C., mais il la place bien loin de ce contexte

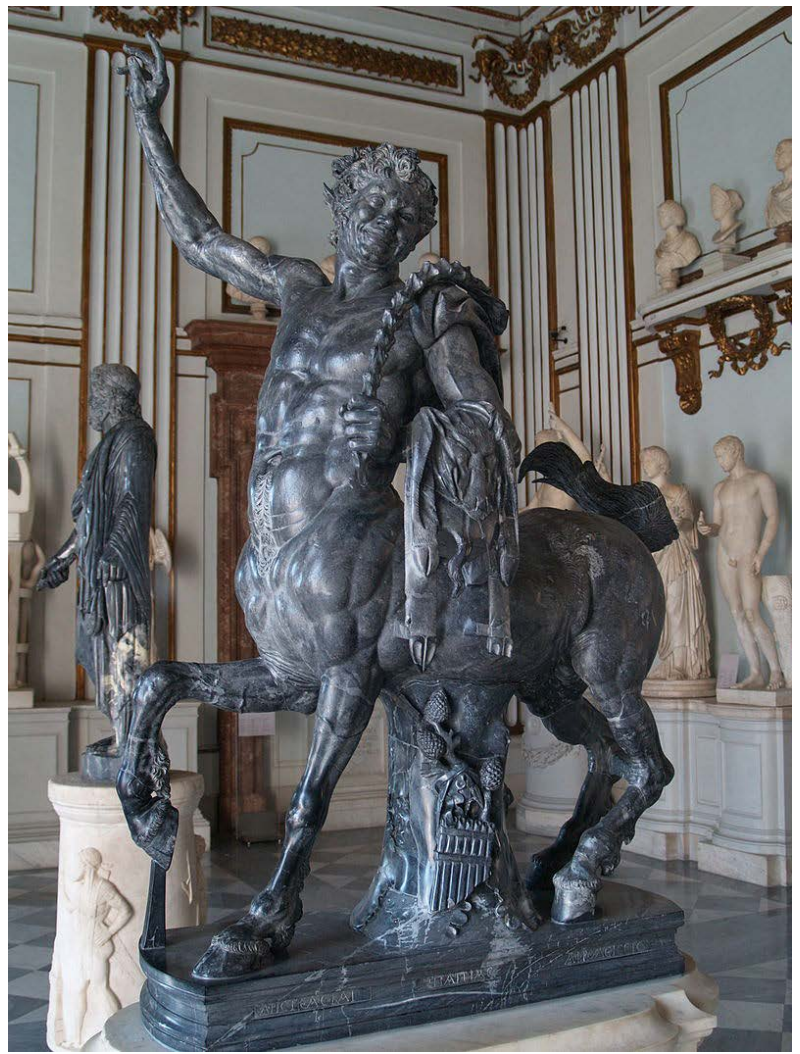

Figure 2. Statue réalisée par Aristeas et Papias, Tivoli, villa d'Hadrien, certainement une copie de la fin du $\mathrm{I}^{\mathrm{er}}$ siècle $-\mathrm{II}^{\mathrm{e}}$ siècle apr. J.-C. d'après des originaux de l'époque hellénistique.

Rome, Musei Capitolini, inv. MC0656. Source : https://commons.wikimedia.org/wiki/File:Centauro_ joven_01.JPG

(Miguel Hermoso Cuesta, CC BY-SA 3.0) querelleur $^{12}$. Le tableau est décrit par Lucien de Samosate au II $^{\mathrm{e}}$ siècle apr. J.-C. ${ }^{13}$, qui ne peut contempler l'œuvre originale, car elle aurait été perdue en mer avec une partie du butin saisi lors du pillage d'Athènes en 86 av. J.-C. Lucien commente donc une copie dont il dit qu'elle est exposée chez un peintre athénien, et 《 réalisée avec une minutieuse exactitude d'après le tableau lui-même. " ${ }^{14}$ Selon l'auteur, le tableau montre une centauresse couchée qui allaite deux nouveau-nés centaures, l'un à ses seins de femme, l'autre à ses mamelles de jument ; au-dessus du groupe, un centaure rieur brandit un lionceau pour effrayer gentiment sa progéniture. L'œuvre de Zeuxis est très éloignée de l'image traditionnelle et contemporaine du centaure. Elle n'est apparemment pas le reflet d'un mythe, car elle ne correspond à aucun récit ancien connu, et parce qu'elle est unique en son genre, au moins jusqu'au II $^{\mathrm{e}}$ siècle apr. J.-C., époque pendant laquelle le sujet sera repris et modifié ${ }^{15}$. Selon le témoignage de Lucien, le peintre a inventé ce sujet, car il lui offrait le moyen de déployer toutes ses compétences artistiques, et lui-même considérait que la prouesse de son œuvre avait bien plus à voir avec la technique et l'habilité de la représentation qu'avec l'originalité du motif : il s'agissait de la figuration très réaliste de la combinaison des deux natures de l'être mixte ${ }^{16}$. D'un point de vue formel, Zeuxis

9 Pline, Histoire naturelle XXXVI, 18 ; Pausanias, I, 28, 2. Padgett 2003, p. 15-17.

10 Jeanmaire 1970, p. 281.

11 Tsiafakis 2003 , p. 74 et 81.

12 Une représentation exceptionnelle sur un pithos à reliefs du viie siècle av. J.-C. montre la gorgone Méduse dotée d'un arrière-train de cheval (Paris, musée du Louvre, inv. CA 795) : https://commons.wikimedia.org/wiki/File:Perseus_Medusa_Louvre_CA795. jpg).

13 Lucien, VI « Zeuxis ou Antiochos », 3-7.

14 Lucien, VI « Zeuxis ou Antiochos », 3.

15 Pesce 1930.

16 Gourmelen 2008, p. 7 ; Pigeaud 2014, p. 186. 
paraît donc être l'inventeur de la femme centaure et des enfants centaures, mais aussi, d'une manière plus générale de la possibilité d'une génération de centaures.

\section{LA CENTAURESSE À L'ÉPOQUE}

IMPÉRIALE : APPARITION ET

DÉVELOPPEMENT

\section{Observations}

$\mathrm{Si}$ les centauresses sont discrètes dans le monde grec, plus à l'ouest un changement important survient à la fin de la République et se développe à l'époque impériale, puisque l'on observe l'existence d'une cinquantaine de représentations de centauresses et trois mentions littéraires pendant cette période.

$\mathrm{Au} \mathrm{IV}^{\mathrm{e}}$ siècle av. J.-C., les centauromachies sont beaucoup moins présentes dans l'art figuratif en Grèce ${ }^{17}$, et les centaures rejoignent le thiase de Dionysos accompagnés par de petits amours, ouvrant la voie à l'imagerie du triomphe dionysiaque romain où centaures, satyres, ménades et erotes se réunissent dans une joyeuse procession ${ }^{18}$. Dans l'entourage de Dionysos/Bacchus, ils sont généralement musiciens et à l'époque hellénistique apparaissent sous la forme du centaure jeune et du centaure âgé (fig. 2). Le corps puissant des centaures convient tout à fait au triomphe de Bacchus, mais il n'est pas l'« animal de trait » exclusif du dieu, puisque son char est bien souvent tiré par des félins, et parfois même des éléphants ${ }^{19}$. Le rôle du sauvage incivilisé qu'endossaient auparavant les centaures s'atténue donc, ce dont témoigne également la littérature alexandrine qui n'évoque la centauromachie thessalienne qu'implicitement et pour prévenir des méfaits de l'abus de la bois$\operatorname{son}^{20}$. Même quand ils ne sont pas montrés dans un environnement bacchique, ils relèvent d'une forte humanisation, ce que montre une mosaïque de centaures au banquet ${ }^{21}$.

C'est à la suite de cette mutation de l'image des centaures dans l'imaginaire des Anciens que des centaures féminins font leur apparition au $\mathrm{I}^{\text {er }}$ siècle av. J.-C. et se développent jusqu'au $\mathrm{III}^{\mathrm{e}}$ siècle. À l'époque impériale, les centauresses sont représentées surtout dans deux contextes iconographiques : domestique et funéraire. Dans la demeure, elles apparaissent comme des personnages constitutifs d'un décor, sous forme de

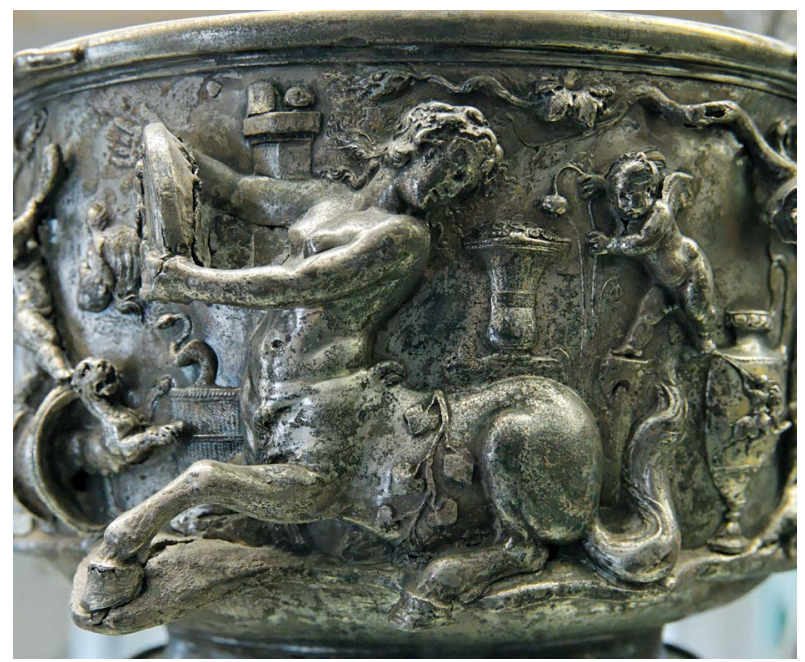

Figure 3. Skyphos dit «Skyphos aux centaures», Berthouville, v. 50 apr. J.C.

Paris, Bibliothèque nationale de France, inv. 56.6. Source : https://commons.wikimedia.org/wiki/ File:Berthouville Centaur skyphos CdM Paris Cat60.jpg (MarieLan Nguyen, CC-BY 2.5)

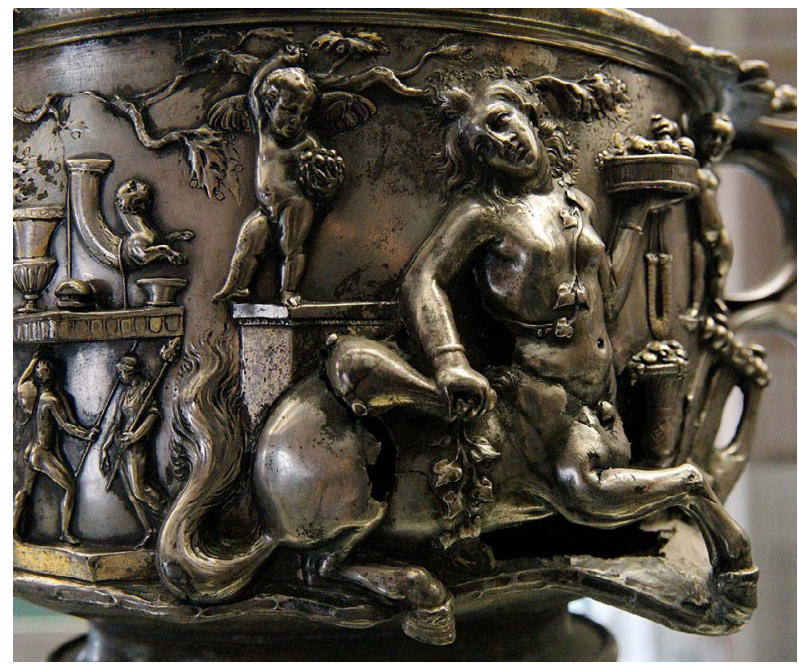

Figure 4. Skyphos dit «Skyphos aux centaures », Berthouville, v. 50 apr. J.C.

Paris, Bibliothèque nationale de France, inv. 56.7.

Source : https://commons.wikimedia.org/wiki/ File:Cup_centaurs_Berthouville_CdM_n2.jpg (MarieLan Nguyen, domaine public)

17 Schörner 2002, p. 122.

18 Grift (Van de) 1984, p. 381 ; Lissarrague 1987, p. 78

19 Boardman 2014, p. 24 et p. 33.

20 Grift (Van de) 1984, p. 382.

21 Wyler 2004, p. 937 : « d'après une aquarelle de G. Marsigli : F. Pesando, dans A. d'Ambrosio et al., Storie di un'eruzione, n.7, p. $252 »$. 


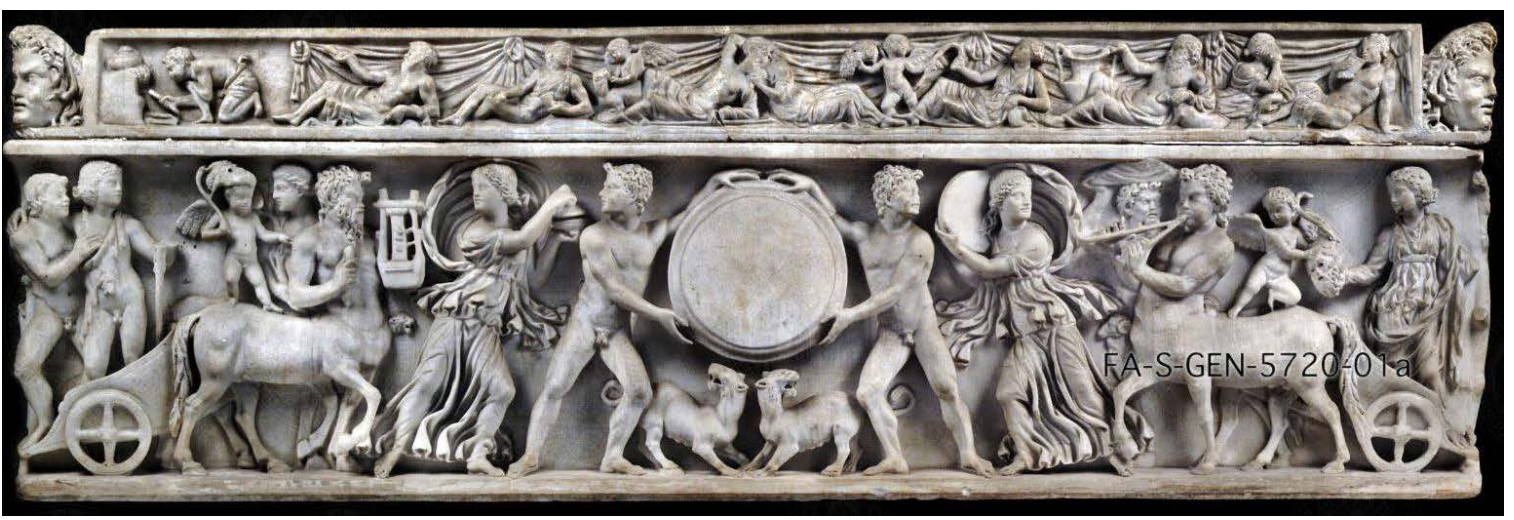

Figure 5. Face principale de sarcophage, Rome, époque antonine.

Vatican, Musei Vaticani. Source : https://arachne.dainst.org/entity/5406935 (Arachne, Gisela Geng, CCBY-NC-ND 3.0)

statuettes, de représentations sur du mobilier (porte-lampes, vases à boire, coffrets), des mosaïques et des fresques. Une intaille et cinq camées représentent une centauresse allaitante ${ }^{22}$ ou Dionysos et Ariane triomphant dans un bige tiré par deux centaures, mâle et femelle ${ }^{23}$. Quant au contexte funéraire, une centauresse est attestée sur un autel ${ }^{24}$, mais ce personnage féminin est surtout présent sur la face principale de sarcophages à partir du $\mathrm{II}^{\mathrm{e}}$ siècle (vingt-trois occurrences). Que ce soit à l'intérieur d'une maison ou sur un sarcophage, les centauresses sont représentées principalement dans un contexte dionysiaque, qui concerne une quarantaine d'occurrences. D'autres contextes narratifs de représentation, moins fréquents, sont ceux de la violence et de la victoire ${ }^{25}$.

Les centauresses sont le plus souvent représentées dans un environnement bacchique, que le dieu soit sur son char, dans le thiase, ou bien que sa présence ne soit qu'évoquée, ce dont témoignent des paires de canthares et de $s k y p h o i^{26}$ (fig. 3 et 4 ) ou des fresques ${ }^{27}$. Les reliefs de sarcophages sont le support de représentation des centauresses le plus fréquent. Sur ces derniers, elles sont presque toujours harnachées au char de Dionysos et accompagnées d'un centaure mâle qui est barbu ou imberbe. Quelques configurations récurrentes ressortent. L'une d'elles figure Dionysos sur son char accompagné du thiase formé de satyres, d'erotes, de ménades, de Pan et parfois d'animaux (panthère, bouc) ${ }^{28}$. Une autre configuration montre deux cortèges bacchiques affrontés (d'un côté Dionysos, de l'autre Ariane ou Sémélé), qui convergent vers un clipeus central (fig. 5) $)^{29}$. Quand ils tirent le bige du dieu, les centaures forment le Kentaurenwagen où la centauresse est soit musicienne, soit porteuse d'un élément végétal. Le Kentaurenwagen de centaures musiciens ou porteurs de canthares et/ou d'éléments végétaux est très répandu sur les sarcophages mais il ne s'agit pas néanmoins d'un type de représentation privilégié de centauresses. La dernière forme figure le mythe de Dionysos et d'Ariane à Naxos, lorsque le dieu arrête sa troupe et découvre la fille de Minos (fig. 6) ${ }^{30}$. Dans cette dernière version, la centauresse est toujours agenouillée face à son petit. Dans ces trois configurations, les centaures sont figurés, car Dionysos est sur un char, leur présence est donc directement liée à ce type de représentation du dieu. La centauresse allaitante (theleia hippokentauros ${ }^{31}$ ) en revanche, est un motif original, car la cen-

22 Intaille en onyx nicolo, époque impériale, Copenhague, Musée Thorwaldsen, inv. 1745 ; camée en onyx, $\mathrm{I}^{\mathrm{er}-\mathrm{II}} \mathrm{e}^{\mathrm{e}}$ siècles, Londres, British Museum, inv. 1867,0507.201.

23 Camées en sardonyx, $\mathrm{I}^{\text {er }}$ siècle av. J.-C., Saint-Pétersbourg, Musée de l'Ermitage, inv. ГР12696 et inv. ГР12669 ; camée en sardonyx, fin du $\mathrm{I}^{\text {er }}$ siècle, Paris, BnF, inv. camée.79; camée en sardonyx, milieu du ${ }^{\text {er }}$ siècle, Naples, Musée archéologique, inv. 25846.

24 Relief funéraire, marbre, 41-50 apr. J.C., Paris, Musée du Louvre, inv. MR 933.

25 Neuf exemples : urne cinéraire, marbre, $\mathrm{III}^{\mathrm{e}}-\mathrm{II}^{\mathrm{e}}$ siècle av. J.-C., Paris, Musée du Louvre, inv. MA2353 ; urne cinéraire, marbre, II ${ }^{\mathrm{e}}$ siècle av. J.-C., Volterra, Musée Guarnacci, inv. 271 ; sarcophage, marbre, milieu du II ${ }^{\mathrm{e}}$ siècle, Ostie, inv. SBAO 16651 ; sarcophage, marbre, début du III ${ }^{e}$ siècle., Cagliari, Musée archéologique, inv. 6141 et 6142 ; mosaïque, époque impériale, Tivoli, Villa d'Hadrien, triclinium/øecus E ; mosaïque, milieu du Iv siècle, Cherchel, Musée de Cherchel ; cratère cantharoïde, 300-290 av. J.-C., Londres, British Museum, inv. 1856,1226.15 ; monnaie, bronze, fin du III siècle, Londres, British Museum, inv. 1850,0601.51.

26 Canthares, argent, milieu du Ir siècle, Naples, Musée archéologique, inv. 25376 et inv. 25377 ; skyphoi, argent, milieu du Ir siècle, Paris, BnF, Cabinet des Médailles et Antiques, inv. 56.6 et 56.7.

27 Fresques, antérieures à 79 apr. J.-C., Naples, Musée archéologique, inv. 9133.

28 Par exemple : sarcophage, marbre, époque sévérienne, Rome, Musée des Thermes ; sarcophage, marbre, époque sévérienne, Philadelphie, Penn Museum, inv. MS4017.

29 Par exemple : sarcophage, marbre, II ${ }^{\mathrm{e}}$ siècle, Marseille, Musée d'archéologie méditerranéenne, inv. 1646 ; sarcophage, marbre, milieu du II ${ }^{\mathrm{e}}$ siècle, Vatican, Palais du Latran, inv. 10425.

30 Par exemple : sarcophage, marbre, époque sévérienne, Vatican, Galerie des Candélabres IV 30 ; sarcophage, marbre, deuxième quart du III ${ }^{\mathrm{e}}$ siècle, Paris, Musée du Louvre, Ma 1346.

31 Pasquier 2011 ; Pesce 1930 ; Schörner 2002. 


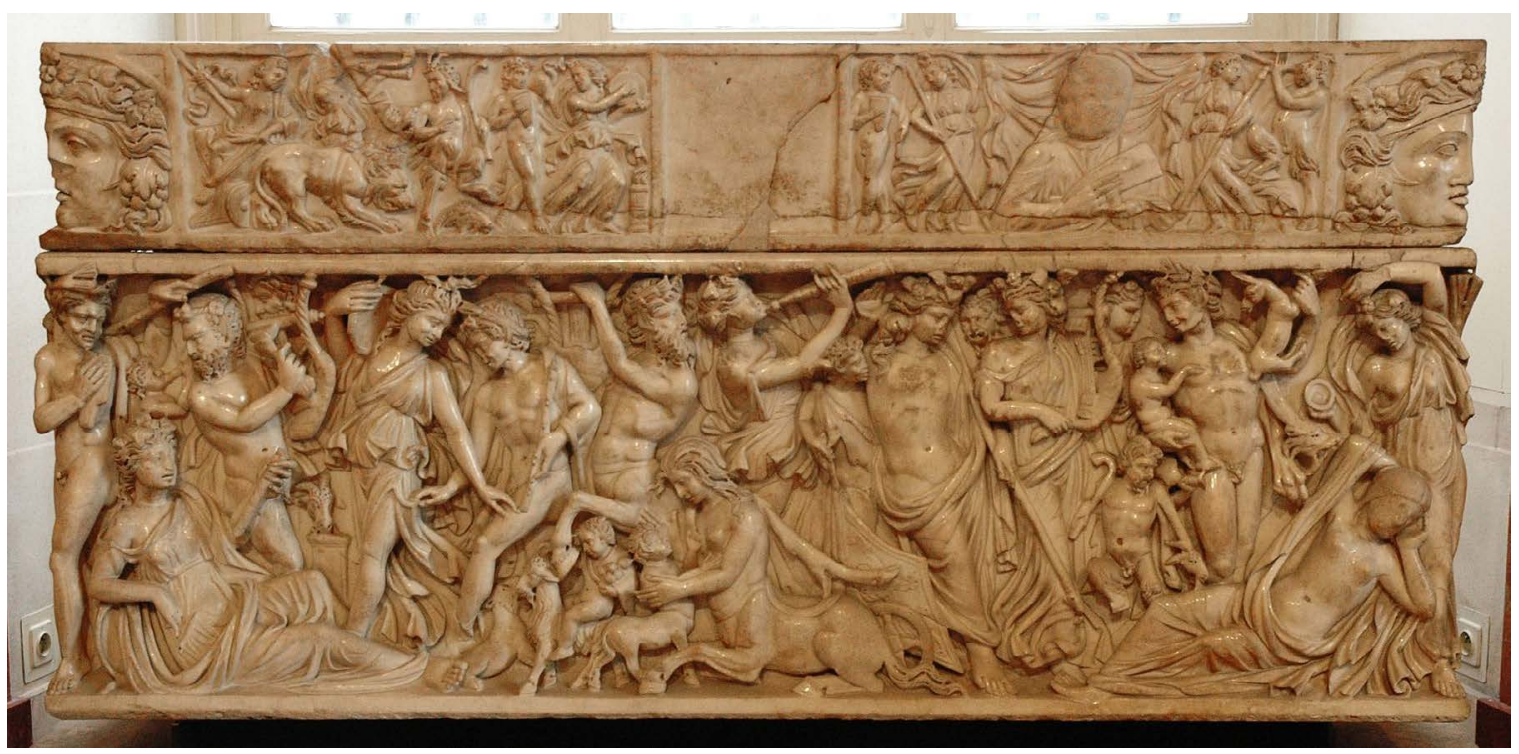

Figure 6. Face principale du sarcophage d'Ariane, Saint-Médard-d'Eyrans, $2^{\mathrm{e}}$ quart du III ${ }^{\mathrm{e}}$ siècle apr. J.-C.

Paris, musée du Louvre, inv. Ma 1346. Source : https://commons.wikimedia.org/wiki/File:Sarcophagus Ariadne_Louvre_Ma1346.jpg (MarieLan Nguyen, domaine public)

tauresse est au premier plan et constitue, avec son petit, un motif indépendant du centaure et même du reste de la composition ${ }^{32}$.

Les statuettes, la vaisselle et les éléments mobiliers présentent presque exclusivement des centauresses dionysiaques. Il est possible que certaines de ces représentations, en apparence isolées ${ }^{33}$, aient formé une paire avec un centaure, constituant alors un couple des deux sexes de l'espèce mythique, ce dont atteste l'exemple des quatre statues-fontaines retrouvées à Oplontis (fig. 7) 3 $^{34}$. Des duos centaure/centauresse se retrouvent encore dans les fresques de la villa « de Cicéron » à Pompéi, où deux centauresses aux attributs dionysiaques, l'une sage et l'autre échevelée, associées à deux centaures, participent au décor mural d'un triclinium ${ }^{35}$. Cette hypothèse peut être mise en parallèle avec le répertoire iconographique des canthares et skyphoi en argent qui cette fois ne montre pas le couple de centaures harnaché au char dionysiaque ${ }^{36}$, mais indépendant de la présence de Dionysos/Bacchus, qui n'est signifiée que par l'environnement bacchique et rustique du décor et par les petits personnages habituels de sa suite (un jeune satyre et des erotes). Le mâle et la femelle sont visuellement dissociés, chacun étant représenté sur une face ; ils ne peuvent jamais être observés en même temps mais « fonctionnent » pourtant ensemble, mettant en scène une opposition par le genre.

Cette répartition du masculin et du féminin illustre implicitement et à un niveau de lecture différent la pensée dionysiaque qui aime jouer sur les contrastes d'humeur (centaure torturé/centaure jovial), d'âge (centaure juvénile/centaure âgé), ici de sexe $\mathrm{e}^{37}$. Ces oppositions peuvent trouver leur source dans la poésie hellénistique qui use des contrastes d'humeur ${ }^{38}$. L'un des objets les plus significatifs de ce contraste est un porte-lampes en bronze du $\mathrm{I}^{\mathrm{er}}$ siècle av. J.C. ou du $\mathrm{I}^{\mathrm{er}}$ siècle apr. J.C., qui réunit des membres féminins des thiases marin et terrestre, avec en bas une centauresse sauvage - en train de cabrer, bras droit tendu vers le haut -, en haut une néréide montée sur un dauphin qui dévore un poulpe $\mathrm{e}^{39}$. Ces deux personnages féminins semblent à première vue opposés, d'une part par leurs attitudes - la néréide est paisiblement assise sur un dauphin tandis que la centauresse est saisie en pleine action -, d'autre part à cause de l'environnement où elles évoluent, marin et terrestre, dont l'association est incongrue. La néréide est empreinte d'une grande « féminité » ou sensualité, présentée à la manière d'une Aphrodite ${ }^{40}$, le corps largement découvert. Or, la centauresse se présente im-

32 Pour une étude précise de ce motif, voir Pesce 1930.

33 Par exemple : protomé, bronze, première moitié du I ${ }^{\text {er }}$ siècle av. J.-C., Berlin, Staatliche Museen, inv. 1967.4.

34 Statues-fontaines, marbre, $\mathrm{I}^{\text {er }}$ siècle av. J.-C., Oplontis, inv. Opl. 55. Pour une étude précise, voir De Caro 1976, p. $205-219$.

35 Naples, musée archéologique, inv. 9133.

36 Voir, n. 25

37 Grift (Van de) 1984, p. 382.

38 Grift (Van de) 1984, p. 383.

39 Paris, Musée du Louvre, inv. Br 4759 (ensemble) et Br 4758 (centauresse). Pasquier 2011. https://art.rmngp.fr/fr/library/artworks/ support-de-lampe-orne-d-une-centauresse_bronze

40 Pasquier 2011, p. 21. 
médiatement aux antipodes de cette « féminité » de la néréide, difficile à appréhender à cause de son hybridité. Néanmoins certains points communs existent entre les deux figures. Le premier se perçoit dans le détail du sac que la centauresse porte en bandoulière sur le torse. Ce sac contient une portée de trois jeunes félins, qui sont sujets à différentes interprétations. Le centaure étant chasseur par excellence, nous pouvons d'abord penser à un butin de chasse. Mais la manière dont elle les porte (elle ne les brandit pas comme c'est le cas ailleurs ${ }^{41}$ ) et la proximité avec la poitrine humaine rappellent davantage une fonction nourricière ${ }^{42}$. Enfin, ce qui semble être la " clé » de cet étonnant assemblage est leur lien avec Dionysos, chacune prenant part à un thiase, l'un marin l'autre terrestre. Cet objet étonnant a pu finalement avoir, selon A. Pasquier, pour vocation le divertissement, l'amusement provoqué par une mise en scène et une association d'éléments absurde donc surprenante $^{43}$. Le groupe de la néréide montée sur un dauphin ne prend pas place sur les flots, mais est perché sur un arbre ; d'un autre côté, le centaure est passé de mâle à femelle et de prédateur à nourrice. La centauresse transgresse ici le caractère traditionnel du centaure pour une expression qui n'est pas « sage », mais qui, par son éventuelle fonction nourricière, rejoint le monde civilisé. Néanmoins, la centauresse ne va pas ici jusqu'à enfanter, elle reste un «monstre »" Les centauresses ne vont en revanche pas jusqu'à retranscrire l'opposition d'âge souvent exprimée entre le centaure jeune et le centaure âgé, qu'illustrent de nombreux sarcophages ${ }^{45}$ et les centaures $« \mathrm{du}$ Capitole » découverts dans la villa d'Hadrien à Tivoli, évoqués plus haut ${ }^{46}$.

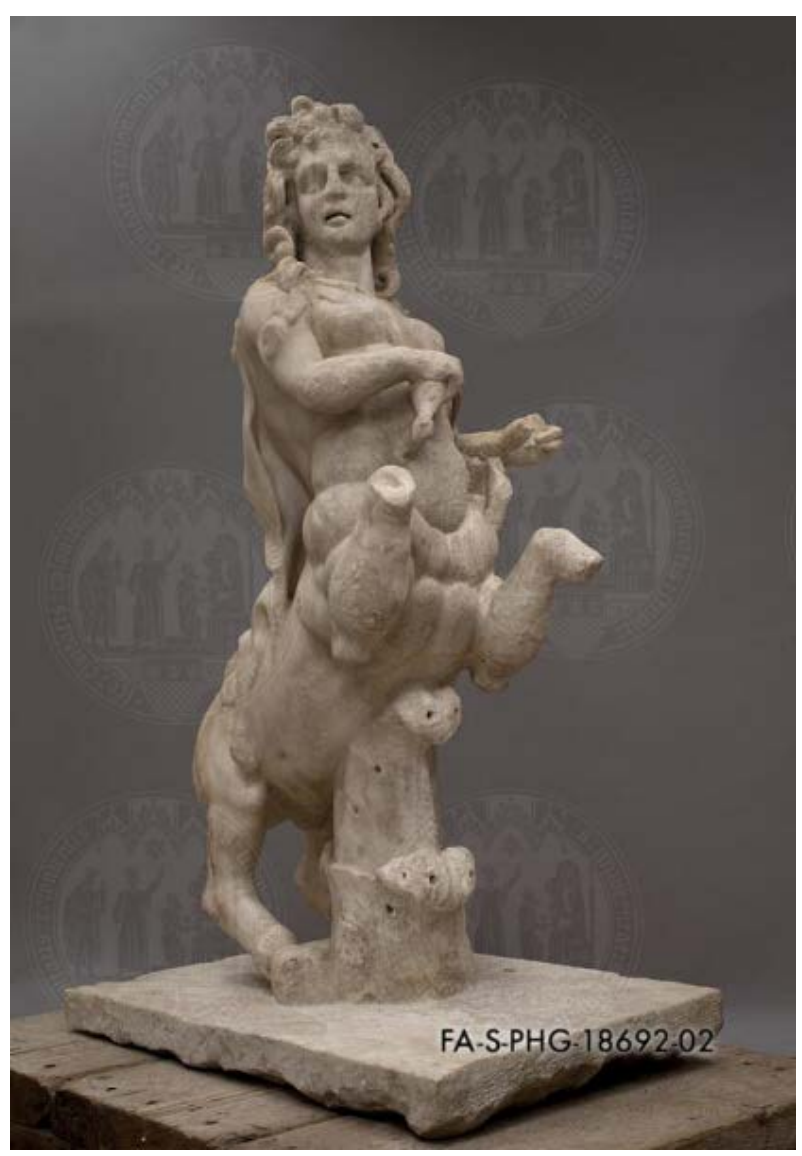

Figure 7. Statue-fontaine, Oplontis (Torre Annunziata), probablement une copie du $\mathrm{I}^{\mathrm{er}}$ siècle apr. J.-C. d'après un original du $\mathrm{II}^{\mathrm{e}}$ siècle av. J.-C. Oplontis, inv. Opl. 71. Source : https://arachne.dainst. org/entity/6868301 (Arachne, Philipp Groß, CC-BYNC-ND 3.0)

\section{Cyllare et Hylonomé, un couple de centaures ovidien}

Seules trois références littéraires mentionnant un centaure féminin nous sont parvenues de l'Antiquité et elles sont relativement tardives, car la première, celle d'Ovide, qui est la seule qui place une centauresse dans un récit, date de la fin du $\mathrm{I}^{\mathrm{er}}$ siècle av. J.C. ou du début du $\mathrm{I}^{\mathrm{er}}$ siècle apr. J.C. Les deux autres sont deux descriptions de tableaux, l'une de l'œuvre de Zeuxis par Lucien au II $^{\mathrm{e}}$ siècle déjà évoquée, et une autre de Philostrate, qui décrit un tableau vu à Naples qui figure une troupe de centauresses et de tout jeunes centaures, en plein air. Ce tableau, s'il a existé, est un autre témoin de la valeur des centauresses comme figure artistique appréciée de l'élite romaine.

Le texte le plus intéressant pour nous est celui des Métamorphoses d'Ovide (XII, 393428) dans laquelle le poète met en scène un couple de centaures, Cyllare et Hylonomé, au cœur du combat contre les Lapithes. Plusieurs détails donnés par l'auteur montrent que Cyllare est un centaure singulier, notamment par sa grande beauté ou le fait qu'il ne partage pas les vices de ses semblables, tels que l'ivrognerie ou les violentes pulsions érotiques. La description de sa compagne Hylonomé la distingue aussi par les soins qu'elle se prodigue afin de parfaire sa beauté (bains, coiffures, parfums, accessoires et vêtements) pour plaire à Cyllare. Les deux

\footnotetext{
41 Un fragment de relief de miroir à boite en bronze de la $2^{\mathrm{e}}$ moitié du IV siècle av. J.C. (Paris, musée du Louvre, inv. Br 4286) et l'une des deux statues-fontaines d'Oplontis (inv. Opl. 71).

42 Pasquier 2011, p. 33.

43 Pasquier 2011, p. 43.

44 De Giorgio et Galtier 2012, p. 13 et p. 16.

45 Par exemple, où figure aussi une centauresse : sarcophage, marbre, milieu du II ${ }^{\mathrm{e}}$ siècle, Vatican, inv. 10425.

46 Rome, Musée du Capitole, inv. 656 et 658.
} 
êtres, par leurs activités, leur apparence et les relations qu'ils entretiennent, paraissent bien plus proches de la civilisation humaine que de la sauvagerie des centaures. De plus, rien n'est dit explicitement concernant la double nature d'Hylonomé, comme si Ovide souhaitait taire ou amoindrir son hybridité. Selon J. B. DeBrohun, le passage serait une digression ayant pour but de ponctuer les scènes sanglantes par une incise qui évoque « un monde de paix et de tendresse domestique »s'inscrivant dans les conventions du récit épique ${ }^{47}$. Ovide insiste en effet sur des concepts éloignés de la guerre (la beauté, l'harmonie, la concorde, l'amour), et cette romance à la fois idyllique et funeste inscrit le passage dans la poésie lyrico-élégiaque ${ }^{48}$. Il paraît alors difficile de considérer l'apparition d'Hylonomé chez Ovide comme la première mythologie d'une centauresse ; il s'agirait plutôt d'une savante manœuvre littéraire à vertu poétique permettant de soulever des questions philosophiques telles que l'opposition entre natura et cultus, l'animal et l'humain, le masculin et

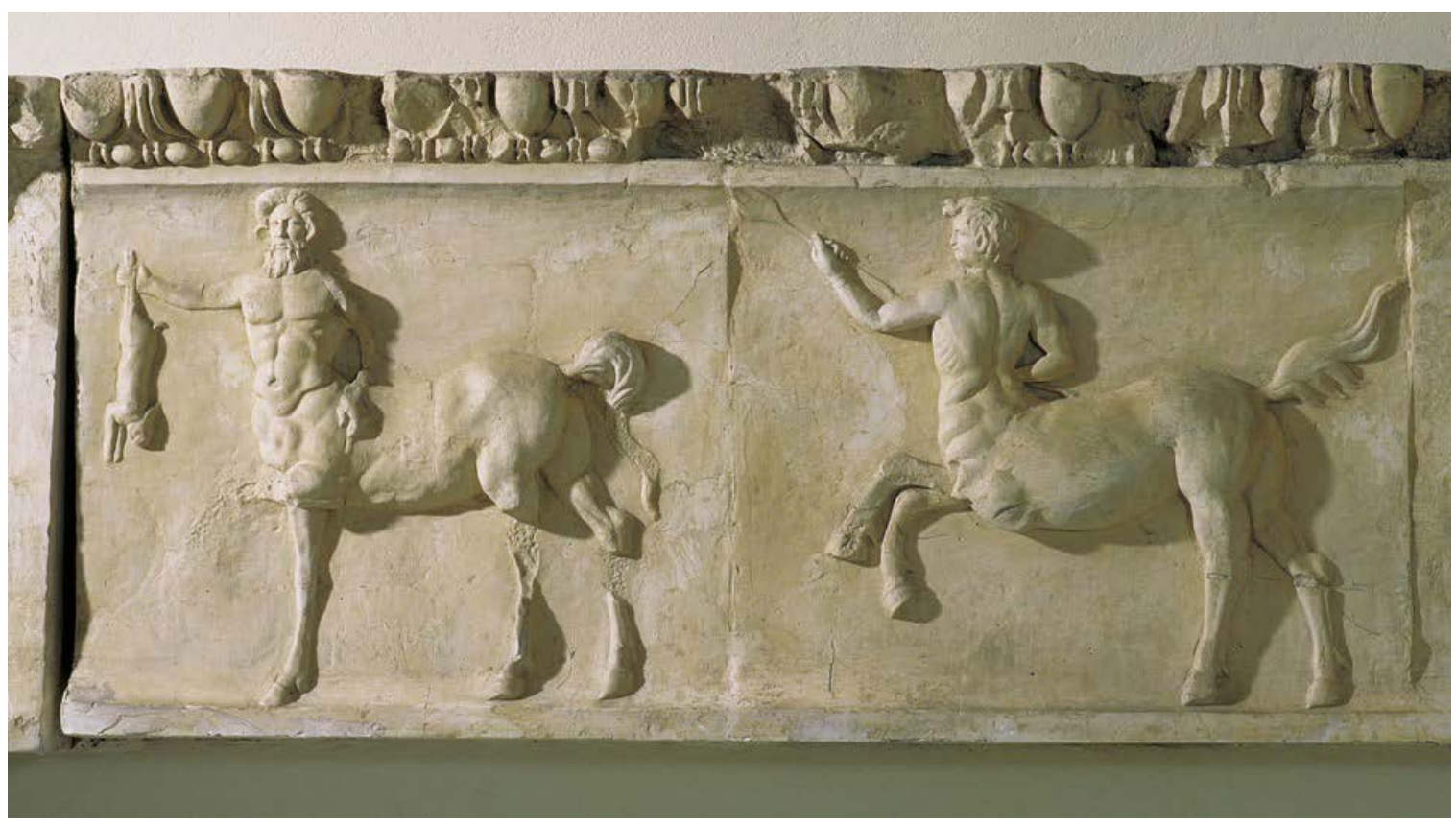

Figure 8. Métopes de la frise de centaures du frons scaenae du théâtre d'Orange, époque augustéenne.

Orange, musée d'art et d'histoire d'Orange. Source : https://commons.wikimedia.org/wiki/File:969_ ORG3002.JPG (Culturespaces, CC-BY-SA 3.0)

le féminin, l'amour et la guerre. Néanmoins, constatons que cette centauresse, la seule qui soit mise en scène dans un mythe littéraire, contraste avec les centaures sauvages « anciens ». Cette différence est en accord avec ce que l'on observe au même moment dans la figuration où les centauresses illustrent un apprivoisement du sauvage.

\section{Un personnage au service de l'idéologie impériale}

Les événements qui ont troublé l'Italie à la fin de la République ont favorisé la croyance d'un retour possible à un nouvel Âge d'Or, pensée encouragée par la poésie hellénistique et la prophétisation de cette nouvelle ère, par exemple dans la tradition mystique orphique ${ }^{49}$. Les thèmes de l'Âge d'Or et du monde dionysiaque ont été rapprochés très tôt, et les Bacchantes d'Euripide ainsi que la race dorée évoquée par Hésiode ont fortement influencé l'imaginaire romain ${ }^{50}$. Bien qu'associée avec l'avènement céleste d'Apollon, cette idée accorde une place majeure à Dionysos dont l'aspect pastoral, l'entente entre les hommes et les animaux sauvages dans sa suite, ainsi que le mythe du démembrement et de la résurrection, conviennent à cette nouvelle ère ${ }^{51}$. Le cadre de cet âge d'or est rustique, bucolique et idyllique, ses acteurs sont aussi bien les hommes que les animaux, car tous les êtres vivants vivent en harmonie. Le tout est empreint d'une grande piété, incarnée à partir de la fin du $\mathrm{I}^{\mathrm{er}}$ siècle av. J.C. dans l'art figuré par les paysages sacro-idylliques ${ }^{52}$. Les

DeBrohun 2004, p. 417

48 DeBrohun 2004, p. 418 sq.

49 Wyler 2012, p. 4.

50 Wyler 2012, p. 3.

51 Wyler 2012, p. 6.

52 Sauron 2007, p. 201. 


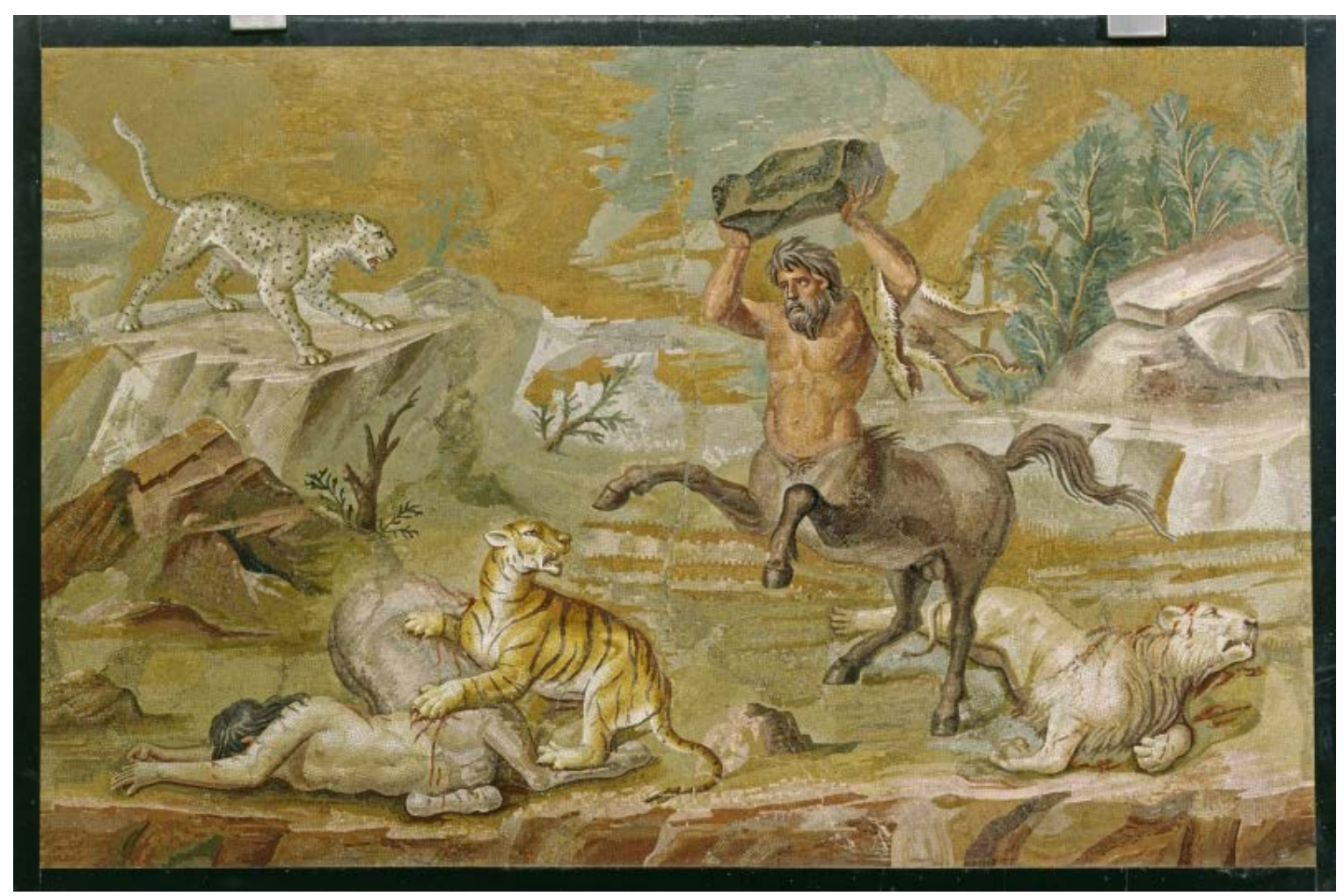

Figure 9. Mosaïque, villa d'Hadrien, Tivoli, v. 120-130 apr. J.-C.

Berlin, Staatliche Museen, inv. Mos. 1. Source : http://www.smb-digital.de/

eMuseumPlus?service $=$ ExternalInterface $\&$ module $=$ collection\&objectId $=697074 \&$ (Antikensammlung der Staatlichen Museen zu Berlin - Preußischer Kulturbesitz, Johannes Laurentius, CC-BY-NC-SA)

bergers renvoient à cette image par leur ruralité, leur aspect traditionnel, ancien et en un sens « originel»; dans l'entourage de Dionysos, les satyres ainsi que Pan font particulièrement référence aux pasteurs, notamment quand ils jouent de l'aulos ou de la syrinx et portent le lagobolon ${ }^{53}$. Cet engouement pour les promesses dionysiaques réservées aux initiés déteint à la fin de la République dans la décoration des maisons de riches citoyens, d'abord en tant qu'ostentation « externe », visible de tous, se retranchant ensuite dans les parties privées des demeures ${ }^{54}$. Ce répertoire figuratif est visible à la fois dans les éléments qui décorent les pièces (fresques, mosaïques, statues) et dans le mobilier usuel domestique (vaisselle, coffret, porte-lampes, etc.), qui pouvait être employé lors de la pratique de banquets, certains réellement utilisés, d'autres seulement exposés. En tant qu'allusions à la culture grecque, ces objets et surtout leur décor permettaient au propriétaire et à ses invités de revendiquer leur philhellénisme, leur raffinement, de stimuler les conversations et d'exposer leur paideia $^{55}$. Les témoins les plus parlants conservés de nos jours sont situés dans la baie de Naples ou à Rome, où de très riches demeures présentent un savant programme décoratif dans lequel l'ornementation, tant celle des murs que des pavements et du mobilier, se déploie harmonieusement dans toute la maison ${ }^{56}$.

Nous l'avons dit, au Ir siècle av. J.C. et par la suite, les centaures sont souvent intégrés au thiase de Dionysos. Hors du contexte privé, ce phénomène est également attesté au service du pouvoir, ce dont témoigne la frise de centaures du théâtre antique d'Orange (fig. 8) ${ }^{57}$. La mise en scène de centaures et de centauresses tantôt musiciens, tantôt porteurs d'animaux pour le sacrifice, les présente dociles et même « auxiliaires ou personnel d'un rituel. ${ }^{58}$ Ici, les centaures apprivoisés renvoient symboliquement à « la célébration de la victoire impériale des forces violentes ou négatives rattachées à ces monstres, tout à fait caractéristique de la mise en images de l'idéologie augustéenne $\aleph^{59}$, faisant évidemment écho à la thématique de l'Âge d'Or, avec de surcroît l'introduction du féminin. Un autre document témoigne de cette domestication extrême du centaure, dans laquelle il a perdu son caractère prédateur : il s'agit d'une mosaïque qui ornait un triclinium de la

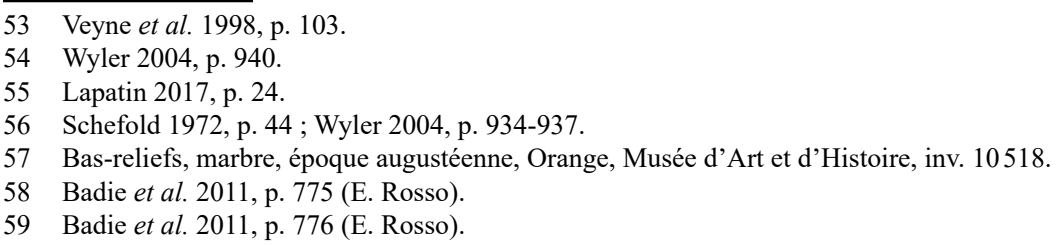


villa d'Hadrien à Tivoli (fig. 9), dont une copie postérieure de moindre qualité a été découverte à Cherchel ${ }^{60}$. Elle figure un couple de centaures dans une zone aride et désertique attaqué par des fauves (lion, tigre). Cette mosaïque de grande qualité, réalisée en opus vermiculatum, s'inscrit dans un ensemble de cinq mosaïques représentant des divinités pastorales, des paysages rustiques et des masques ${ }^{61}$. La centauresse est au sol le flanc ensanglanté, tandis que son compagnon s'apprête à assommer son agresseur. Cet exemple est représentatif du changement considérable de l'image du centaure à partir de l'époque impériale qui même en pleine nature, son environnement de prédilection, a perdu tout caractère agressif et prédateur et se trouve victime d'une attaque d'animaux sauvages qu'il incarnait auparavant. La centauresse n'est pas montrée hargneuse et combattante comme le sont traditionnellement les centaures, au contraire elle semble vulnérable. L'intégration de cette scène dans le programme décoratif de la villa d'un empereur témoigne de la portée du sujet : l'hybride auparavant monstrueux est désormais dompté, plus encore, il est inclus dans le monde civilisé et est soumis aux mêmes dangers. À partir du $\mathrm{I}^{\mathrm{er}}$ siècle av. J.C., la centauresse participe à la réduction du côté monstrueux des centaures, processus engagé depuis qu'ils ont rejoint le thiase ${ }^{62}$, et le pousse à l'extrême puisque s'ouvre la voie de la reproduction, ce dont témoigne le thème de la centauresse allaitante. L'hybride qu'est le centaure n'est plus un monstre ou un contre-exemple de l'humanité, mais intègre la civilisation.

\section{CONCLUSION}

L'étude des attestations de centauresses a montré une concentration des occurrences à l'époque romaine. La pensée d'un nouvel Âge d'Or, favorisée par la fin des guerres civiles et le philhellénisme qui touche les élites romaines aux II $^{\mathrm{e}}$ et $\mathrm{I}^{\mathrm{er}}$ siècles av. J.C., est promue par l'idéologie augustéenne et a fortement participé à l'exaltation des décors dionysiaques dans les demeures. Les centaures, hybrides monstrueux, demeuraient à l'époque classique des êtres isolés à la fois des hommes et des dieux, à qui les mythes n'offraient pas même l'accès à la reproduction. Ils incarnaient à ce titre et de la même manière que les satyres ${ }^{63}$ l'altérité sauvage opposée à la civilisation grecque ${ }^{64}$. Mais ce rôle perd peu à peu de son importance, et, on l'a dit, les centaures s'assagissent notamment en rejoignant le thiase de Dionysos. Dans le contexte de la fin de la République et du début de l'Empire, l'apparition franche de centaures « au féminin » s'inscrit dans l'idée de la domestication du sauvage et dans la pensée d'un Âge d'Or où hommes et animaux vivent en harmonie et en paix. Dans le cadre de cette évolution, il semblerait que les artistes aient voulu dépasser la frontière qui existait entre l'homme et l'animal à travers la figure hybride du centaure en l'intégrant au monde civilisé, notamment par la création d'une altérité féminine, les centauresses, modifiant ainsi la tradition mythique selon laquelle les centaures étaient des monstres stériles. En introduisant une parèdre, l'espèce mythique du centaure a la possibilité de procréer, d'avoir une descendance, ce qui la rend plus acceptable, car proche des êtres vivants non mythiques. Il rejoint ainsi la civilisation, et les artistes vont même parfois jusqu'à amoindrir voire taire son caractère hybride, tel que le poète Ovide.

Les mêmes constats sont possibles avec l'évolution du dieu Pan et dans une moindre mesure des satyres qui, à la fin de la République et jusqu'au III $^{\mathrm{e}}$ siècle, apparaissent sous une forme féminine ${ }^{65}$. Pour finir, citons $\mathrm{S}$. Wyler qui écrit à propos des satyres de la mégalographie de la villa « des Mystères » que « ces figures hybrides (...) sont le cœur de la logique de l'Âge d'Or dionysiaque, et la poussent aussi à son extrême. $»^{66}$ Outre la domestication du sauvage, c'est aussi l'idée d'un renouvellement générationnel, d'une renaissance harmonieuse qui est signifiée, et les mixanthrôpoi monstrueux intensifient l'aspect harmonieux de ce monde rêvé.

60 Mosaïque, 120-130, Berlin, Staatliche Museen, inv. Mos. 1.

61 Adembri 2000, p. 68. Les quatre mosaïques sont conservées à Rome, Musei Vaticani.

62 Schörner 2002, p. 120.

63 Lissarrague 1990, p. 66.

64 Schörner 2002, p. 114.

65 Quantin 2000.

66 Wyler 2012, p. 9. 


\section{BiBLIOGRAPHIE}

\section{Liste des abréviations}

CNRTL, Centre National de Ressources Textuelles et Lexicales.

\section{Sources anciennes}

Ovide, Métamorphoses, XII.

Lucien de Samosate, Eikones, VI, « Zeuxis ou Antiochos ».

Philostrate, La galerie des tableaux.

\section{Travaux}

Adembri B. 2000, La Villa d'Hadrien, Milan.

Aston E. 2011, « Mixanthrôpoi: animal-human hybrid deities in Greek religion », Kernos supplement 25, Liège.

Badie A., Moretti J.C., Rosso E. et TARdy D. 2011, « L'ornementation de la frons scaenae du théâtre d'Orange : l'élévation de la zone centrale », in T. Nogales et I. Rodà (éd.), Roma y las provincias : modelo y diffusión, ome, p. 193-202.

Boardman J. 2014, The Triumph of Dionysos. Convivial processions, from Antiquity to the present day, Oxford.

CAlame C. 2015, Qu'est-ce que la mythologie grecque?, Paris.

DeBrohun J.B. 2004, « Centaurs in Love and War: Cyllarus and Hylonome in Ovid 'Metamorphoses' 12.393428 », The American Journal of Philology 125, 3, p. 417-452.

De Caro S. 1976, « Sculture della villa 'di Poppea' in Oplontis », Cronache Pompeiane II, p. 184-225.

De Giorgio J.P. et Galtier F. (éd.) 2012, Le monstre et sa lignée, Paris.

Gourmelen L. 2008, « La Centauresse de Zeuxis. $\mathrm{Du}$ bon usage de l'insolite en peinture et en littérature ", in A. Bouloumié (dir.), Écritures insolites : Cahier XXXIII, Angers, disponible sur : https://books.openedition.org/pur/12117 [consulté en $12 / 2019]$.

GRIFT (Van De) J. 1984, «Tears and Revel: the Allegory of the Berthouville Centaur Scyphi », American Journal of Archeology 8833, p. 377-388.

Jeanmaire H. 1970, Dionysos, histoire du culte de Bacchus, Paris.

LAPATIN K. 2017, « Le luxe romain de la maison à la tombe et au sanctuaire », in M. Avisseau-Broustet et C. Colonna, Le luxe dans l'Antiquité. Trésors de la Bibliothèque nationale de France, Gand, p. 16-27.

LisSARRAGUE F. 1987, « De la sexualité des Satyres », Mètis. Anthropologie des mondes grecs anciens 21, p. 63-90.

LisSARRAGUe F. 1990, « The Sexual Life of Satyrs », in D.M. Halperin, J.J. Winkler et F.I. Zeitlin (éd.), Before Sexuality: The Construction of Erotic Experience in the Ancient Greek World, Princeton, p. 53-66.

Padgett J.M. 2003, « Horse Men: Centaurs and Satyrs in Early Greek Art », in J.M. Padgett (éd.), The Centaur's smile: the human animal in early Greek art (exhibition, Princeton University Art Museum, October 11, 2003 - January 18, 2004; Museum of Fine Arts, Houston, February 22 May 6, 2004), Princeton, New Haven, Londres, p. 3-46.

PASQuier A. 2011, « De la néréide à la centauresse. Un porte-lampes en bronze inédit conservé au musée du Louvre », Monuments et mémoires de la Fondation Eugène Piot 90, p. 5-43.

Pesce G. 1930, « La Theleia Hippokentauros in gemme e sarcofagi romani », Bulletino della Commissionne Archeologica Communale di Rome LVIII, p. 57-104.

Pigeaud J. 2014, « Lucien ou l'ekphrasis », in S. Dubel (éd.), Lucien de Samosate. Portrait du sophiste en amateur d'art, Études de littérature ancienne 22, Paris, p. 177-210. 
QuAntin F. 2000, « Le dieu Pan au féminin à Bouthrôtos : une influence italienne ? », in É. Déniaux (éd.), Le canal d'Otrante et la Méditerranée antique et médiévale, Bari, p. 67-79.

SAURON G. 2007, La peinture allégorique à Pompéi : le regard de Cicéron, Paris.

SCHEFOLD K. 1972, La peinture pompéienne. Essai sur l'évolution de sa signification. Latomus 108 , Bruxelles.

SCHÖRNER G. 2002, « H theleia hippokentauros des Zeuxis - Familiariseung des Fremden ?», Boreas 25, p. 97-124.

TSIAFAKIS D. 2003, « 'ПE $\Lambda \Omega P A ’$ Fabulous Creatures and/or Demons of Death? », in J.M. Padgett (éd.), The Centaur's smile: the human animal in early Greek art (exhibition, Princeton University Art Museum, October 11, 2003 - January 18, 2004 ; Museum of Fine Arts, Houston, February 22 May 16, 2004), Princeton, p. 73-104.
VIAL H. 2012, « Filiation, monstruosité et métamorphose dans les Métamorphoses d'Ovide », in J.-P. De Giorgio et F. Galtier (éd.), Le monstre et sa lignée, p. 65-82.

Veyne P., Lissarrague F. et FrontisiDucroux F. 1998, Les mystères du gynécée, Paris.

WyLER S. 2004, “"Dionysos domesticus”. Les motifs dionysiaques dans les maisons pompéiennes et romaines ( $\mathrm{II}^{\mathrm{e}}$ siècle avant $-\mathrm{I}^{\mathrm{er}}$ siècle après J.C.) ", Mélanges de l'École française de Rome. Antiquité 116/2, p. 933-951.

Wyler S. 2012, « Dionysiaca aurea : the development of Dionysiac images from Augustus to Nero », Neronia electronica 2, p. 319, disponible sur : http://www.sien-neron.fr/2013/02/neroniaelectronica-\%e2\%80\%93-fascicule-2-2012/ [consulté en 06/2019]. 